\title{
Impacto de la aplicación de la Ley 13059 de Eficiencia Energética en relación a la nueva Ordenanza de Usos del Suelo de la ciudad de La Plata y la Norma IRAM 11900 de Etiquetado de Edificios
}

Impact of the aplication of Energy Efficiency 13059 Act in relation to the new Land Use Ordinance of the city of La Plata and the Building Labeling Standard IRAM 11900

\section{Carolina Soledad Vagge J orge Daniel Czajkowski}

\section{Resumen}

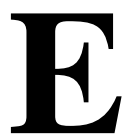

1 crecimiento urbano introduce permanente presión a las áreas metropolitanas. En países periféricos la demanda de energía crece a mayor velocidad que el crecimiento poblacional y la oferta, creando crisis y escasez. El objetivo principal de este trabajo es prever el impacto que tendrá la Ley 13059 de Eficiencia Energética en relación a la nueva Ordenanza de Usos del Suelo de la ciudad de La Plata y la Norma de Etiquetado de Edificios (IRAM 11900). Esta norma establece una metodología simplificada para el cálculo del nivel de eficiencia energética de las envolventes de los edificios susceptibles de ser calefaccionados. Todavía, es evidente que existe una importante falta de adecuación entre los objetivos teóricos formulados para el desarrollo de la ciudad y las conflictivas estrategias técnicas propuestas para satisfacerlos. En cuanto a la aplicación de la ley de eficiencia energética al modelo de edificio de construcción usual en La Plata, no verifica sus valores de transmitancia térmica K. Tampoco verifica el riesgo de condensación ni el coeficiente global de pérdidas G. Del mismo modo, cuando se aplica el protocolo de etiquetado de eficiencia energética que plantea la norma IRAM 11900, el edifico clasifica como $\mathrm{H}$. Se puede ver que el impacto de ahorro potencial en la demanda de energía anual para calefacción, si se comienza a implementar la Ley de Eficiencia Energética sería de un $46 \%$.

Palabras-clave: Uso racional de energía. Densidad edilicia. Calidad ambiental. Paisaje urbano.

Carolina Soledad Vagge Laboratorio de Arquitectura y Hábitat Sustentable,-Facultad de Arquitectura y Urbanismo

Universidad Nacional de La Plata Calle 47, 162

1900

La Plata - Buenos Aires - Argentina Te.: +54 (221) 423-6587 Int. 255 E-mail: carolinavagge@yahoo.com.ar

J orge Daniel Czajkowski Laboratorio de Arquitectura y Hábitat Sustentable,-Facultad de Arquitectura y Urbanismo

Universidad Nacional de La Plata E-mail: czajko@yahoo.com

Recebido em 27/07/11 Aceito em 21/05/12

\section{Abstract}

Urban growth produces constant pressure on metropolitan areas. In developing countries the energy demand is growing at a faster pace than the population and energy supply, generating crisis and scarcity. The main goal of this paper is to forecast the impact of Energy Efficiency Act 13059 with regards to the new Land Use Ordinance of the city of La Plata and the Building Labeling Standard IRAM 11900. This standard provides a simplified methodology for calculating the level of energy efficiency of building envelopes that can be heated. However, there is a significant lack of fit between the theoretical objectives formulated for the development of the city and the conflicting technical strategies proposed to achieve them. As for the enforcement of energy efficiency laws in the usual building model in La Plata, it does not check their thermal transmittance values of $K$, nor does it check the risk of condensation or global loss coefficient G. Similarly, when applying the energy efficiency labeling protocol established by IRAM 11900, the building classifies as $H$. One can see that the impact of potential savings in annual energy demand for heating would be 46\%, if the Energy Efficiency Act were implemented.

Keywords: Rational use of energy. Building density. Environmental quality. Urban landscape. 


\section{Introducción}

El trabajo de investigación presentado se encuadra dentro de la realización de una beca doctoral tipo II CONICET para aspirar al grado de doctora en Arquitectura y Urbanismo de la FAU-UNLP. El tema de trabajo refiere a un "Modelo alternativo de planeamiento estratégico energético-ambiental de áreas metropolitanas MAPES basado en diferenciales de tejido urbano". También se enmarca dentro del proyecto de investigación PICT ANPCyT 2006-956: "Eficiencia energética edilicia en áreas metropolitanas. Evaluaciones mediante auditorias y propuestas de estándares".

De acuerdo con la División de Población de las Naciones Unidas, Latinoamérica es la región en vías de desarrollo más urbanizada del mundo. En la actualidad la región se encuentra más urbanizada que ciertas partes del mundo desarrollado y se espera que el porcentaje de la población que habita en las ciudades en Latinoamérica continúe aumentando, por lo que para el año 2030 se estima que la cifra alcance el $86 \%$.

El rápido aumento de la población en las ciudades ha tenido implicaciones económicas, políticas y sociales. (SIEMENS, 2011).

De cara al futuro los desafíos medioambientales en las áreas urbanas de Latinoamérica crecerán. Los expertos predicen que las ciudades, especialmente las de tamaño mediano, como La Plata, incrementarán tanto su área como su población. Este nuevo espacio urbano ya está invadiendo un terreno ambientalmente marginal y la infraestructura se verá bajo una presión cada vez mayor debido al incremento poblacional y los efectos extremos del cambio climático. Adicionalmente, el crecimiento de ciudades por fuera del marco formal de planificación también continuará. Abordar estos desafíos requerirá una visión amplia a largo plazo y, por eso, el propósito de este trabajo es poder construir estrategias y mejores prácticas que ayuden a las ciudades a adoptar una visión que les permita alcanzar la sustentabilidad ambiental y mejorar la eficiencia energética de sus edificios.

Las ciudades latinoamericanas no realizan un muy buen papel en la construcción de edificios ecológicamente responsables. Existen varios obstáculos como lo son las políticas cortoplacistas y la descentralización. Los proveedores de servicios trabajan de manera aislada, lo cual es tremendamente desfavorable al enfoque integral que se requiere para hacer las ciudades más sustentables (SIEMENS, 2011).
El arquitecto Norman Foster sostiene que los edificios deben ser flexibles y capaces de adaptarse a los cambios. La mejor solución de proyecto integra los aspectos sociales, tecnológicos, estéticos, económicos y ambientales.

La ubicación y función de un edificio, su flexibilidad y su previsión de vida útil, su orientación, su forma y estructura, sus sistemas de calefacción y ventilación y los materiales utilizados suponen un impacto sobre la cantidad de energía necesaria para la construcción, el funcionamiento y el mantenimiento.

La arquitectura sustentable no tiene que ver únicamente con el diseño de edificios individuales. Hay una relación directa entre densidad urbana y consumo de energía. Las ciudades con mucha densidad liberan la mejora de calidad de vida cuando la vivienda, el trabajo y los servicios están cerca unos de otros.

En efecto el ciclo imparable de derribar y volver a construir ejerce una presión muy fuerte sobre los recursos naturales y la utilización de energía, en términos de sustentabilidad, la acción de derribar lo ya construido debería ser el último recurso. (FOSTER, 2010)

\section{Situación de referencia}

La Plata es una ciudad fundada como capital de la provincia de Buenos Aires, a finales del siglo XIX, a $60 \mathrm{~km}$. de la capital de la República Argentina. $\mathrm{Su}$ plan territorial estaba compuesto por tres elementos: la ciudad o casco urbano, siguiendo la teoría urbana arquitectónicas higienistas y estéticas de la época, el área productiva rural y el puerto sobre el Río de La Plata.

Como otras ciudades Argentinas y latinoamericanas el Casco y la ciudad nacieron juntos y predeterminados desde un modelo ideal (ETULAIN; LÓPEZ, 1997).

Ese modelo de ciudad comenzó a declinar en las últimas décadas debido a que el gran crecimiento demográfico no fue acompañado por un proyecto de planificación urbana integral. El aumento poblacional trajo asentamientos marginales, un mayor número de edificios en el casco urbano, más vehículos, más contaminación, saturación de los servicios públicos y falta de preservación del patrimonio histórico.

Geográficamente pertenece a la región pampeana, siendo sus coordenadas: latitud: $-35,0$ longitud: 57,9 y altitud: 15 m. s.n.m. 
Las variables climáticas principales de la zona son: TBS min. med. Anual $=12^{\circ} \mathrm{C}$; TBS max. med. Anual $=21,5^{\circ} \mathrm{C}$; HR media anual $=79 \%$; en verano la temperatura máxima de diseño $=31,4^{\circ} \mathrm{C}$ y en invierno la mínima de diseño $=1,0^{\circ} \mathrm{C}$; GD anuales de calefacción, (base $\left.18^{\circ} \mathrm{C}\right)=1178^{\circ} \mathrm{D}$, GD anuales de enfriamiento, (base $\left.23^{\circ} \mathrm{C}\right)=274^{\circ} \mathrm{D}$. (CZAJKOWSKI; GÓMEZ, 2002).

\section{Ley 13059 de acondicionamiento térmico de edificios}

La finalidad de la Ley es establecer las condiciones de acondicionamiento térmico exigibles en la construcción de los edificios, para contribuir a una mejor calidad de vida de la población y a la disminución del impacto ambiental a través del uso racional de la energía. $\mathrm{Su}$ alcance son todas las construcciones públicas y privadas destinadas al uso humano (viviendas, escuelas, industrias, hospitales, entre otras) que se construyan en el territorio de la provincia de Buenos Aires, siendo los municipios la Autoridad de Aplicación de la Ley. A partir de la sanción de esta Ley serán de aplicación obligatoria normas técnicas del Instituto de Racionalización de Materiales (IRAM) referidas a acondicionamiento térmico de edificios y ventanas, en su edición más reciente.

En cuanto a las disposiciones de diseño la ley exige verificar:

(a) la Transmitancia Térmica " $\mathrm{K}$ " $\left(\mathrm{W} / \mathrm{m}^{2} . \mathrm{K}\right)$, su cálculo se realiza utilizando el método y los valores normalizados de Resistencias Térmicas y Conductividades Térmicas " $\lambda$ " (W/m.K), indicados en la Norma IRAM 11601 (INSTITUTO..., 1996a) y empleando la guía para la aplicación de la misma. Verifica los puentes térmicos;

(b) los Riesgos de Condensación según las Normas IRAM 11625 (INSTITUTO..., 2000a) y 11630 (INSTITUTO..., 2000b) que, tanto las temperaturas superficiales como las intersticiales en los muros, techos y pisos no sean igual o inferiores en ningún caso a las correspondientes temperaturas de rocío, tanto en la superficie como en todo el espesor del paramento, sea éste homogéneo o heterogéneo;

(c) el coeficiente volumétrico de pérdida de calor $\mathrm{G}_{\text {cal }}$ establecido en la Norma IRAM 11604

(INSTITUTO..., 2001c), a fin de alcanzar un ahorro de energía en calefacción en las edificaciones y facilitar el planeamiento y gestión energética ambiental del hábitat de la Provincia de Buenos Aires; y (d) la calidad térmica de ventanas en cuanto a infiltración de aire y transmitancia térmica establecidas en la Norma IRAM 11507-1 y $11507-$ 4 (INSTITUTO..., 2001a; 2001b).

\section{Propuesta del municipio}

La Plata cuenta con nuevo Código de Edificación. La flamante ordenanza, aprobada el 28 de abril del 2010 por el Concejo Deliberante, reemplaza una norma vigente desde hace 47 años.

Según se subraya, el nuevo Código de Edificación no sólo reorganiza y depura los parámetros vigentes, sino propone cambios conceptuales, incorpora innovaciones en el campo de la gestión de permisos, la seguridad edilicia y las responsabilidades de particulares, empresas y profesionales en las diferentes etapas de ejecución de las obras.

En el año 2000 se aprobó un Código de Planeamiento Urbano (MINICIPALIDAD..., 2010). Después de cuatro años de debate entre diferentes actores institucionales y profesionales, que integraron el Consejo de Ordenamiento Urbano y Territorial (COUT), creado en 1996 como espacio para analizar, debatir y generar propuestas de mejoramiento de la ciudad.

En la reciente reforma del Código, por el contrario, no fue respetada la ordenanza que exige la intervención del COUT. Sólo se convocó a las cámaras constructoras, a ciertas empresas inmobiliarias $y$ a un reducido grupo de especialistas, generando un fuerte repudio de parte de distintos sectores que no fueron llamados y que criticaron la premura por votar la norma y sus objetivos.

El Código de Ordenamiento Urbano aprobado a fines de abril estipula que los edificios que pretendan alcanzar las alturas máximas deberán ubicarse sobre avenidas, diagonales, plazas y parques. Además establece el ensanche del corredor industrial y comercial. En tanto, se delimitan tres anillos en el casco urbano (Figura 1). En el primero, se podrán construir edificios con un máximo de 10 pisos pero con posibilidades de obtener "premios" de hasta 4 pisos más por la construcción de cocheras. En el segundo, se podrán construir 8 pisos con posibilidad de acceder a otros 2 por premios. En tanto, en el tercero, la nueva norma permite construir solamente planta baja y dos pisos sin posibilidad de ampliación.

También, se podrá construir en altura sobre el Eje Fundacional del trazado de la ciudad y se establece una zona de preservación patrimonial. 

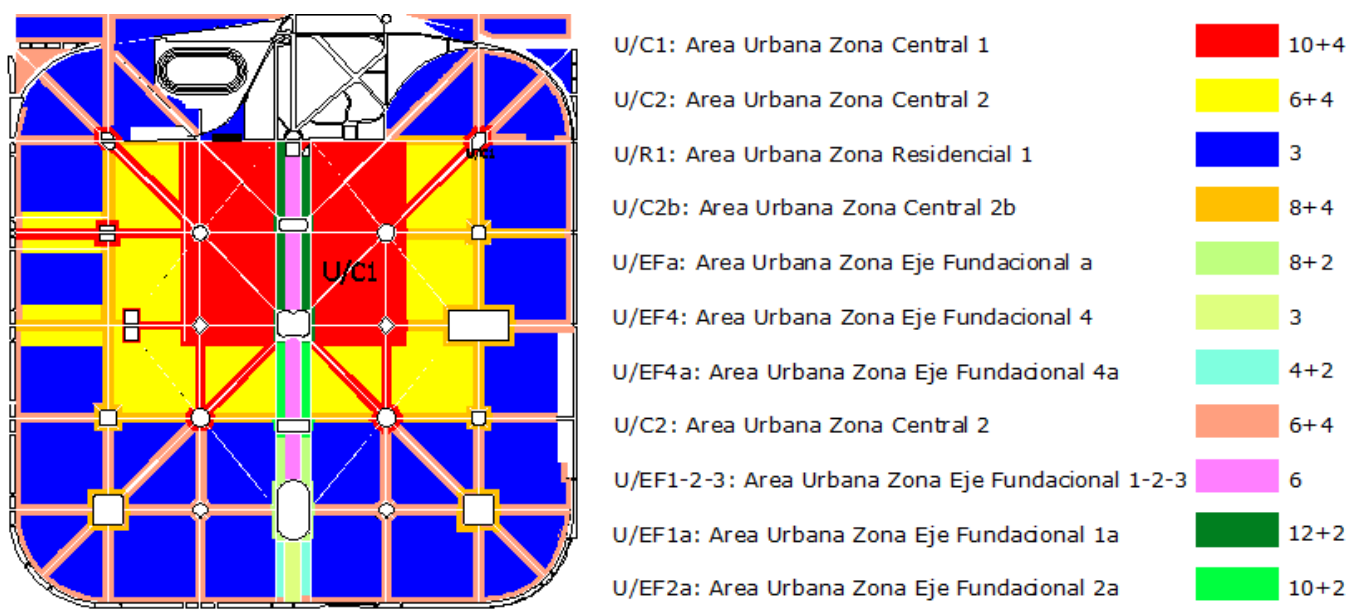

Figura 1- Esquema de alturas máximas permitidas según Indicadores Urbanos del Código de Ordenamiento Territorial y Usos del Suelo

Fuente: propuesto por la Municipalidad de la Ciudad de La Plata (2010).

Nota: en tabla 1 se especifican zonas.

Un aspecto conflictivo, fue la posibilidad instalar clubes de campo y countries en zonas que actualmente corresponden a la producción frutihortícola. Finalmente, las áreas destinadas a estas construcciones se redujeron a $28,72 \mathrm{Km}^{2}$, mientras que con la anterior normativa, se permitía que ocuparan hasta un total de $40,09 \mathrm{Km}^{2}$.

\section{Etiqueta de eficiencia energética de calefacción para edificios: clasificación según la transmitancia térmica de la envolvente (Norma IRAM 11900)}

A partir de 2009 en que se aprobó la Norma IRAM 11900 (INSTITUTO..., 2000c), Argentina cuenta con un protocolo e indicadores de etiquetado de edificios a fin de regular la eficiencia energética en calefacción.

La etiqueta de eficiencia energética especificada en esta norma tiene por objeto informar al consumidor sobre la eficiencia térmica de la envolvente de los edificios, de acuerdo con los parámetros y valores especificados en esta norma.

Mediante la etiqueta se califica la eficiencia a través de un sistema comparativo, compuesto por ocho clases de eficiencia energética identificadas por las letras A, B, C, D, E, F, G y H, donde la letra A se adjudica a las envolventes de los edificios más eficientes y la $\mathrm{H}$ a las menos eficientes.

Esta norma establece una metodología simplificada para el cálculo del nivel de eficiencia energética de las envolventes de los edificios susceptibles de ser calefaccionados, y las características de la etiqueta de eficiencia energética.

El indicador se denomina $\tau$ (tau) y resulta de la diferencia de temperatura ponderada entre una temperatura interior de confort de $20^{\circ} \mathrm{C}$ y las temperaturas superficiales interiores de muros, techos, ventanas, puertas, tabiques y pisos en contacto con el aire exterior. Este indicador se define por la Ecuación 1:

$\tau_{m}=\frac{\sum\left(\tau_{i} \cdot S_{i}\right)}{\sum S_{i}}$

Ec. 1

Siendo:

$\tau_{i}=0,13 \frac{m^{2} \cdot K}{W} \cdot K_{i} \cdot \Delta t\left({ }^{\circ} \mathrm{C}\right)$

$\Delta t$ la diferencia de temperatura de diseño in-terior y exterior $\left({ }^{\circ} \mathrm{C}\right)$;

La resistencia térmica superficial interior $\left(R_{s i}\right)$ se establece en $0,13 \frac{\mathrm{m}^{2} \cdot K}{W}$, a los efectos de la aplicación de la norma 11900 (INSTITUTO..., 2000c); y

$K_{i}$ la transmitancia térmica, que según la IRAM 11601 se establece como $\frac{1}{R_{t}}\left(\mathrm{~W} / \mathrm{m}^{2} \mathrm{~K}\right)$.

$$
K_{i}=\frac{1}{R_{s e}+\sum \frac{e_{j}}{\lambda_{j}}+R_{S i}+\sum R_{c j}}
$$

Siendo:

$R_{s e}$ y $R_{s i}$ las resistencias térmicas superficiales exterior e interior respectivamente. Se obtienen de la IRAM $11601\left(\mathrm{~m}^{2} . \mathrm{K} / \mathrm{W}\right)$;

$e_{\mathrm{j}}$ el espesor de la capa en consideración (m); 
$\lambda_{j}$ la conductividad térmica de la capa en consideración, se obtiene de la IRAM 11601

(INSTITUTO..., 1996a), (W/mK);

$R_{c j}$ la resistencia térmica de las cámaras de aire, en caso de existir, se obtiene de la IRAM 11601 (INSTITUTO..., 1996a) $\left(\mathrm{m}^{2} . \mathrm{K} / \mathrm{W}\right) ; \mathrm{y}$

La transmitancia térmica media ponderada $\left(K_{m}^{\prime}\right)$ en watt por metro cuadrado kelvin, se determina con la siguiente expresión (Ec. 3).

$$
K_{m}^{\prime}=\frac{\sum_{1}^{n}\left(K_{i} \cdot S_{i}\right)}{\sum_{1}^{n} S_{i}}
$$

Siendo:

$K_{i}$ la transmitancia térmica, que en la IRAM 11601 (INSTITUTO..., 1996a) queda establecida como

$$
1 / R_{t}\left(\mathrm{~W} / \mathrm{m}^{2} \mathrm{~K}\right) ; \mathrm{y}
$$

$\mathrm{S}_{\mathrm{i}}$ La superficie de cada una de las componentes de la envolvente $\left(\mathrm{m}^{2}\right)$.

De la interpretación de las ecuaciones 1 a 3 se entiende que la Norma IRAM 11900 (INSTITUTO..., 2000c) no propone en sus 8 categorías valores mínimos o máximos de transmitancias térmicas para la envolvente. Tampoco incorpora la permeabilidad al aire de puertas y ventanas. Solo establece una escala y valores que pueden alcanzarse mediante un adecuado equilibrio entre superficies vidriadas y opacas junto a sus transmitancias térmicas. El nivel más bajo $\mathrm{H}$ permite que prácticamente todo lo que se construye en el presente en Argentina lo integre. El cumplimiento del nivel más exigente A tiende a contemplar los edificios de baja energía.

\section{Objetivo}

El objetivo principal de este trabajo es poder prever el impacto que tendrá la aplicación de la Ley 13059 (ARGENTINA, 2010) de acondicionamiento térmico de edificios en relación al nuevo Código de Ordenamiento Urbano de la ciudad de La Plata y la Norma de Etiquetado de Edificios (IRAM 11900 (INSTITUTO..., 2000c)).

\section{Metodología}

Como caso de estudio se tomo un edificio en la ciudad de La Plata, con las características de los edificios en altura que se están construyendo en la ciudad, en la zona centro de la ciudad UCl (Tabla 1), caracterizada por el nuevo código como el ámbito de centralidad donde se promueve preservar las características significativas ambientales y culturales. Se caracteriza con usos institucionales administrativos de alcance regional, comerciales y residenciales. Se promueve una elevada intensidad de ocupación del suelo $\mathrm{y}$ multiplicidad de actividades. Sus indicadores permiten construir hasta 10 niveles, un Factor de Ocupación del Suelo (FOS) de 0,6, un Factor de Ocupación Total (FOT) de 2,5 y una densidad residencial de $1000 \mathrm{hab} / \mathrm{ha}$. Compensaciones urbanísticas por provisión de cocheras, en este caso permite un $70 \%$ más de FOT y densidad. (Tabla 1)

Como hipótesis de trabajo se considera que el modelo de edificio (Figura 2) tiene uno de sus muros medianeros opaco y compartido con un edificio vecino.

La superficie del terreno es de $400 \mathrm{~m}^{2}$ y la superficie cubierta del edificio: $1470 \mathrm{~m}^{2}$. El modelo de edificio tiene 10 niveles, 3 departamentos por piso de 1 dormitorio cada uno, y en la plata baja un local comercial. Las características técnicas del edificio: la estructura es independiente de hormigón armado, bases, vigas de fundación, columnas, vigas y losas. Su mampostería es de ladrillos huecos y compuesto a la cal con aislamiento hidrófugo. Los revoques son, grueso a la cal y enlucido en yeso, exterior azotado hidrófugo y revoque grueso fratasado a la cal. Los cielorrasos son de yeso aplicado o placas de roca de yeso en todos los ambientes.

\begin{tabular}{|c|c|c|c|c|c|c|c|c|c|c|}
\hline & \multirow{2}{*}{\multicolumn{3}{|c|}{ CARACTERIZACIÓN DE ZONAS E INDICADORES }} & \multirow{2}{*}{ FOS } & \multirow{2}{*}{ NIVELES } & \multicolumn{2}{|c|}{ FOT } & \multicolumn{2}{|c|}{ DENDIDAD } & \multirow{2}{*}{ PREMIOS } \\
\hline & & & & & & Comercial & Residencial & Comercial & \begin{tabular}{|l|} 
Residencial \\
\end{tabular} & \\
\hline \multirow{11}{*}{ 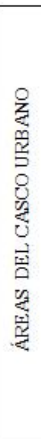 } & \multirow{6}{*}{$\mathrm{U} / \mathrm{EF}$} & Tramo 1 & & \multirow{4}{*}{ 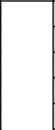 } & \begin{tabular}{|c|}
6 \\
12 \\
\end{tabular} & \multirow{4}{*}{3} & \multirow{4}{*}{2,5} & \multirow{4}{*}{2000} & \multirow{4}{*}{1000} & \multirow[t]{4}{*}{$70 \%$ en FOT y } \\
\hline & & \multirow{2}{*}{ Tramo 2} & & & 6 & & & & & \\
\hline & & & $\mathrm{a}$ & & \begin{tabular}{|l|}
10 \\
\end{tabular} & & & & & \\
\hline & & Tramo 3 & & & $\frac{6}{8}$ & & & & & \\
\hline & & \multirow[b]{2}{*}{ Tramo 4} & & \multirow[b]{2}{*}{0,6} & 3 & 1,6 & 1,2 & 800 & 600 & \\
\hline & & & a & & 4 & 2 & 1.4 & 1000 & 800 & $\begin{array}{l}70 \% \text { FOT y } \\
\text { DENSDDAD }\end{array}$ \\
\hline & $\mathrm{U} / \mathrm{Cl}$ & Area Central, residencial central & & 0,6 & 10 & 3 & 2,5 & 2000 & 1000 & $\begin{array}{l}70 \% \text { FOT y } \\
\text { DENSDAD }\end{array}$ \\
\hline & \multirow{2}{*}{$\mathrm{U} / \mathrm{C} 2$} & \multirow{2}{*}{ Área pericentral } & $\mathrm{U} / \mathrm{C} 2$ & \multirow[b]{2}{*}{0,6} & 6 & \multirow[b]{2}{*}{2,7} & \multirow[b]{2}{*}{2,25} & \multirow[b]{2}{*}{1800} & \multirow[b]{2}{*}{900} & $50 \%$ FOT y \\
\hline & & & $\mathrm{U} / \mathrm{C} 2 \mathrm{~b}$ & & 8 & & & & & DENSIDAD \\
\hline & $\mathrm{U} / \mathrm{C} 3$ & \begin{tabular}{|c|} 
Areas de promoción y Corredores de acceso \\
Principal y Complementarios
\end{tabular} & & 0,6 & 6 & 2,4 & 2 & 1600 & 800 & $\begin{array}{l}30 \% \text { FOT y } \\
\text { DENSIDAD }\end{array}$ \\
\hline & $\mathrm{U} / \mathrm{R} 1$ & Área Residencial del Casco Fundacional & & 0.6 & 3 & & 1.2 & & 100 & \\
\hline
\end{tabular}

\section{Tabla 1 - Indicadores propuestos por la Municipalidad de la Ciudad de La Plata}



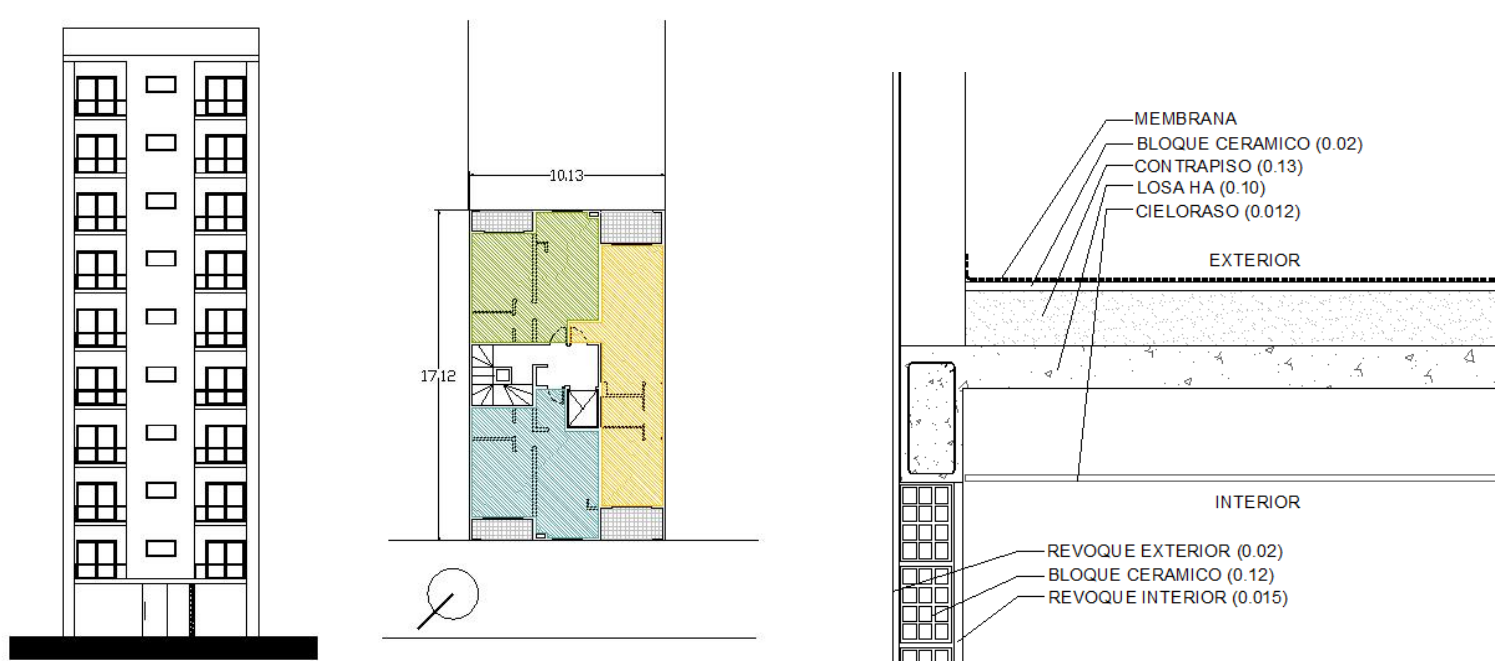

Figura 2 - Vista y planta tipo del modelo de edificio analizado

Nota: detalle constructivo del encuentro del muro y la cubierta.

Los muros interiores y cielorrasos están pintados con látex para interior. Muros exteriores en látex acrílico. La fachada del edificio con revestimiento plástico, tipo "Miksa" acabado texturado. Carpintería: Puertas ventana con marcos de aluminio, hojas de aluminio corredizas vidriadas. En todos los ambientes los pisos son cerámicos, al igual que el revestimiento de los baños.

Al modelo se le aplicó la Ley 13059, verificando su transmitancia térmica $K$, el riesgo de condensación con el programa CEEMACON (GONZALO, 1998) y el coeficiente volumétrico de perdidas térmicas en calefacción $G$.

Se realizó el cálculo de emisiones de Gases de Efecto Invernadero utilizando la calculadora de GEI de Equivalencias de la EPA (Environmental Protection Agency) (ENVIRONMENT..., 2012). Dado que la calculadora EPA corresponde a la matriz energética de EEUU se usó el factor de conversión para adecuar los resultados a la matriz energética argentina para la generación de energía eléctrica principalmente gas natural, combustibles líquidos, hidroelectricidad y nuclear (GONZALEZ, 2010).

Por último, se realizó el cálculo estimativo de la superficie y volumen edificado de la manzana en la actualidad. Se calculó una densidad estimada y luego se determinó una demanda de energía.

Luego se realizaron las mismas estimaciones para un crecimiento de la manzana en un $50 \%$ y luego ya densificada a $1000 \mathrm{hab} / \mathrm{ha}$ con la demanda de energía. Con estos datos se realizaron distintos gráficos para obtener la demanda potencial de energía en calefacción, sin tener en cuenta la Ley, e implementándola. Para de este modo poder inferir el ahorro potencial de energía.
Para integrar las ecuaciones de las curvas de los gráficos, se utilizó el programa Maple. 15.

\section{Resultados}

\section{Aplicación de la Ley 13059 (ARGENTINA, 2010)}

\section{Cálculo de coeficiente de trasmitancia térmica $K\left(W / m^{2} \cdot K\right)$}

$\mathrm{Su}$ cálculo se realizó utilizando el método y los valores normalizados de resistencias térmicas $\mathrm{y}$ conductividades térmicas $\lambda(\mathrm{W} / \mathrm{m} . \mathrm{K})$, indicados en la Norma IRAM 11601 (INSTITUTO..., 1996a) y empleando la guía para la aplicación de la misma.

Se utilizó una planilla de cálculo para verificar el coeficiente de trasmitancia térmica $K$ para cada componente de la envolvente, (IRAM 11601 (INSTITUTO..., 1996a)), tanto para condición de verano como de invierno.

La norma IRAM 11605 (INSTITUTO..., 1996b) propone valores admisibles de $K$ conociendo la temperatura de diseño y la zona bioambiental, para invierno y verano. Propone tres niveles de aislamiento térmico. El decreto 1030/10 (ARGENTINA, 2010) exige el Nivel B y la realidad muestra que solo se alcanza a cumplir el nivel C. Para cada localidad del país en función de la temperatura mínima de diseño exterior y 18, 20 o $22^{\circ} \mathrm{C}$ en el interior de locales se busca evitar el riesgo de condensación superficial interior.

En el caso de los muros el $K$ calculado es de es $1,94 \mathrm{~W} / \mathrm{m}^{2} \mathrm{~K}$ es mayor a 1,85 y menor a 2,00 , por lo tanto cumple con norma nivel $C$ de verano (Figura 3). 
El valor de $\mathrm{K}$ de techos de proyecto es de 2,36 $\mathrm{W} / \mathrm{m}^{2} \mathrm{~K}$, es mayor a los $\mathrm{K}$ admisible según la norma para el nivel $C$, de verano y de invierno (Figura 3).

\section{Verificación del riesgo de condensación intersticial}

A los fines de aplicación de la ley se verificó la arista superior, el encuentro entre el muro y la losa, según lo establecido en la Norma IRAM 11630

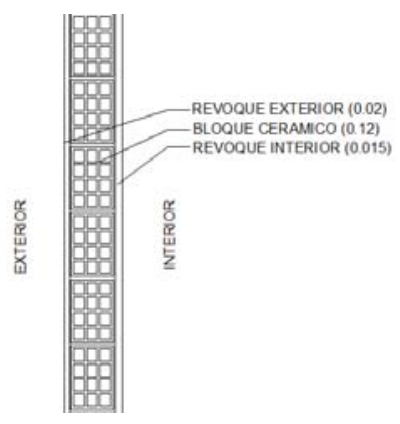

(INSTITUTO..., 2000b), mediante el programa CEEMACON (GONZALO, 1998).

Como se puede observar en la Figura 4, no verifica, el muro condensa a nivel superficial e intersticial.

Figura 3 - Detalle de soluciones constructivas usuales en muros y techos en la actualidad

Nota: Leyenda: transmitancias térmicas usuales:

$$
\begin{aligned}
& \mathrm{K}_{\text {MURO }}=1,94 \mathrm{~W} / \mathrm{m}^{2} \mathrm{~K} ; \mathrm{y} \\
& \mathrm{K}_{\text {TECHO }}=2,36 \mathrm{~W} / \mathrm{m}^{2} \mathrm{~K} .
\end{aligned}
$$

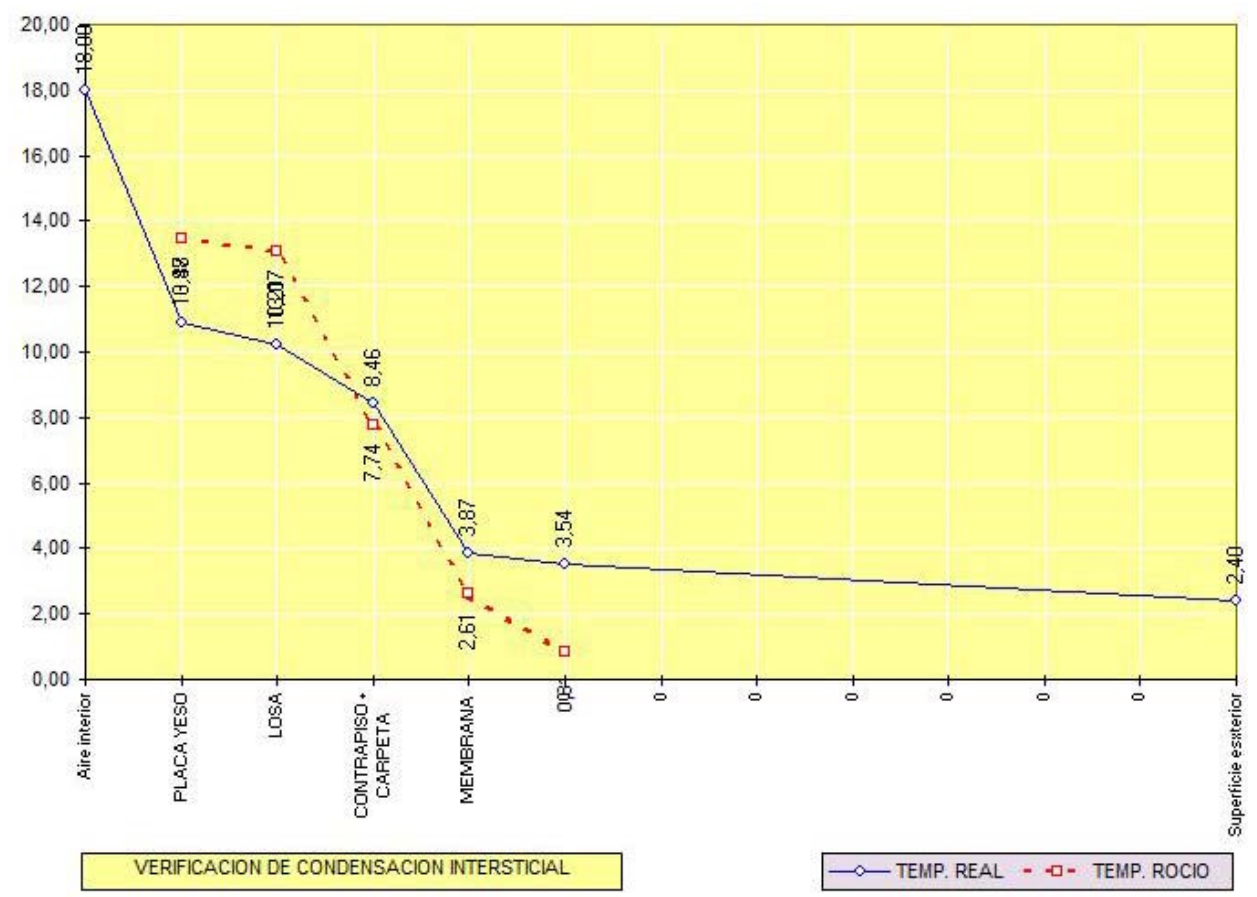

Figura 4 - Gráfico que muestra la condensación en la arista superior del local 


\section{Cálculo coeficiente global de pérdidas G}

Según el protocolo de cálculo de la norma IRAM 11604 (INSTITUTO..., 2001c) se calculó el Coeficiente volumétrico $\mathrm{G}$ de pérdidas de calor:

$G_{C A L}=\frac{\sum K_{m} \cdot S_{m}+\sum K_{v} \cdot S_{v}+\sum \gamma \cdot K_{r} \cdot S_{r}+\text { Per. } P_{p}}{V o l}+0,35 . n$

Ec. 4

Siendo:

$G_{c a l}$ Coeficiente volumétrico de pérdida de calor del edificio de vivienda;

$K_{m}$ la transmitancia térmica de cada uno de los elementos que componen los cerramientos opacos que lindan con el exterior $\left(\mathrm{W} / \mathrm{m}^{2} \mathrm{~K}\right)$;

$S_{m}$ área interior de los cerramientos opacos $\left(\mathrm{m}^{2}\right)$;

$K_{v}$ la transmitancia térmica de cada uno de los elementos que componen los cerramientos no opacos que lindan con el exterior $\left(\mathrm{W} / \mathrm{m}^{2} \mathrm{~K}\right)$;

$S_{v}$ área interior de los cerramientos no opacos $\left(\mathrm{m}^{2}\right)$; $\gamma K_{r}$ transmitancia térmica corregida de cada uno de los elementos que componen los cerramientos opcacos y no opacos que linden con locales no calefaccionados $\left(\mathrm{W} / \mathrm{m}^{2} \mathrm{~K}\right)$;

$S_{r}$ area interior de los cerramientos opacos y no opacos que linden con locales no calefaccionados $\left(\mathrm{m}^{2}\right)$;

Per perimetro del piso en contacto con el aire exterior (m);

$\mathrm{Pp}$ las perdidas por piso en contacto con el aire exterior $\left(\mathrm{W} / \mathrm{m}^{2} \mathrm{~K}\right)$;

Vol volumen interior del edificio de vivienda calefaccionado $\left(\mathrm{m}^{3}\right)$.

0.35 la capacidad especifica del aire del aire; $y$

$n$ el número de renovaciones de aire promedio por hora.

Obtenido el $\mathrm{G}_{\text {cal }}$ de proyecto (Ec. 4): $1,88 \mathrm{~W} / \mathrm{m}^{3}$ ${ }^{\circ} \mathrm{C}$, encontramos que el $\mathrm{G}_{\mathrm{adm}}$ correspondiente a 1178 Grados Día y $3675 \mathrm{~m}^{3}$ es de $1,55 \mathrm{~W} / \mathrm{m}^{30} \mathrm{C}$ (Tabla 2). Realizando la comparación, se puede ver que el $\mathrm{G}$ calculado de proyecto es mayor que el $\mathrm{G}$ admisible, por lo tanto no cumple con los standares de calidad térmica de la norma IRAM 11604 (INSTITUTO..., 2001c).

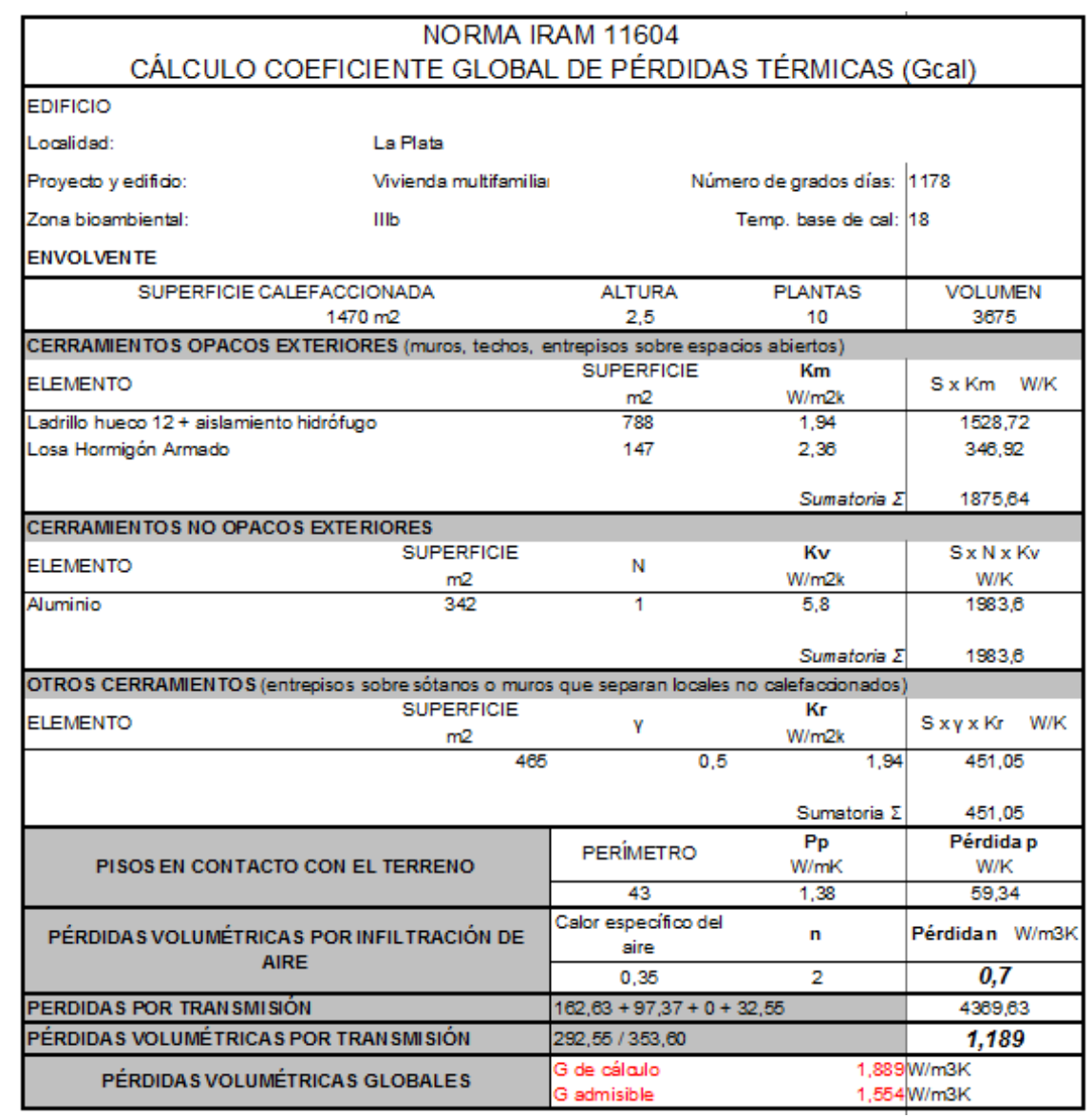

Tabla 2 - Planilla de cálculo del Coeficiente Global de pérdidas térmicas G

Fonte: IRAM 11604 (INSTITUTO..., 2001c). 


\section{Carga térmica anual en calefacción}

La carga térmica anual en calefacción para mantener el total de horas en que se encuentra la temperatura exterior por debajo del confort establecido $\left(18^{\circ} \mathrm{C}\right)$ será de: $\mathrm{Q}_{\mathrm{cal}}=195969$ $\mathrm{kWh} / \mathrm{año}$. Esto se determina mediante la Ec. 5 y los datos de la Tabla 3.

$Q_{\text {cal }}$ anual $=\frac{N \cdot G D_{c a l}\left(18^{\circ} \mathrm{C}\right) \cdot G_{c a l} \cdot V o l}{1000}$

Siendo:

$\mathrm{N}$ número de horas diarias en calefacción (24 hs);

$\mathrm{GD}_{\text {cal }}$ Grados día de calefacción;

$\mathrm{G}_{\text {cal }}$ Coeficiente volumétrico de pérdidas de calor en $\left(\mathrm{W} / \mathrm{m}^{3 \circ} \mathrm{C}\right)$; $\mathrm{y}$

Vol volumen a calefaccionar en $\left(\mathrm{m}^{3}\right)$.

\section{Emisiones $\mathrm{CO}_{2}$}

Para realizar el cálculo de emisiones de Gases de Efecto Invernadero se utilizó la Calculadora de GEI Equivalencias de la EPA (Environmental Protection Agency) (ENVIRONMENT..., 2012). Se determinó que el modelo edilicio emitiría $73 \mathrm{Tn}$ de $\mathrm{CO}_{2}$ al año. Dado que la calculadora EPA corresponde a la matriz energética de EEUU se usó el factor de conversión para adecuar los resultados a la matriz energética argentina para la generación de energía eléctrica principalmente gas natural, combustibles líquidos, hidroelectricidad y nuclear (GONZALEZ, 2010).

Emisiones modelo edilicio $=$ Tn/año $\mathrm{CO}_{2}$.

Sup. total a construir del modelo de edificio $=1470$ $\mathrm{m}^{2}$;

Emisiones Hoy $\approx 0,49 \mathrm{Tn} / \mathrm{m}^{2}$ año $\mathrm{CO}_{2}$;

Superficie total a construir por manzana $\approx 32250$ $\mathrm{m}^{2}$;

$\mathrm{UC} 1 \approx 144$ manzanas;

$\mathrm{UC} 1 \approx 4644000 \mathrm{~m}^{2}$;

$\mathrm{UC1} \approx 227556$ Tn/año $\mathrm{CO}_{2} ; \mathrm{y}$

$\mathrm{UC1}_{\text {Ley }} \approx 122880 \mathrm{Tn} /$ año $\mathrm{CO}_{2}$.
La reducción de emisiones solo para el área microcentro de la ciudad sería de 104675 Tn/año $\mathrm{CO}_{2}$ solamente en demanda anual de energía en calefacción.

\section{Etiquetado energético de edificios}

El objetivo de la Norma IRAM 11900 (INSTITUTO..., 2000c) es establecer una metodología simplificada para el cálculo del nivel de eficiencia energética de las envolventes de los edificios susceptibles de ser calefaccionados, y las características de la etiqueta de eficiencia energética.

La etiqueta de eficiencia energética tiene por objeto informar al consumidor sobre la eficiencia térmica de la envolvente de los edificios, de acuerdo con los parámetros y valores especificados en esta norma.

Mediante la etiqueta se califica la eficiencia a través de un sistema comparativo, compuesto por ocho clases de eficiencia energética identificadas por las letras A, B, C, D, E, F, G y H, donde la letra A se adjudica a las envolventes de los edificios más eficientes y la $\mathrm{H}$ a las menos eficientes. En el caso de estudio, dada las características técnicas del Edificio de vivienda multifamiliar la etiqueta que le corresponde es $\mathrm{H}$ (Tabla 4 y Figura 5).

\section{Situación actual y escenario futuro}

En la Figura 6 se puede observar el paisaje cotidiano de la ciudad de La Plata. Muchas de las viviendas unifamiliares fundacionales fueron y son demolidas para levantar nuevos edificios en altura. En la Figura 7 se puede ver una volumetría de la manzana actual donde se está construyendo el edificio analizado, y su posible evolución a mediano plazo teniendo en cuenta los indicadores de la nueva ordenanza. Se pasaría de una densidad actual estimada en $290 \mathrm{hab} / \mathrm{ha}$ a $1000 \mathrm{hab} / \mathrm{ha}$. Debido a los reclamos de vecinos y ONG desde hace un año se vienen realizando fuertes inversiones para mejorar la provisión de agua potable y energía eléctrica de toda la zona bajo fuerte crecimiento.

Tabla 3 - Tabla de datos para calcular la carga térmica anual en calefacción

\begin{tabular}{c|c|c|c}
\hline $\mathbf{N}(\mathbf{h s})$ & Gdcal & Gcal $\left(\mathbf{W} / \mathbf{m 3}^{\circ} \mathbf{C}\right)$ & Vol ${\left(\mathbf{m}^{3}\right)}$ \\
\hline 24 & 1178 & 1,88 & 3675 \\
\hline
\end{tabular}




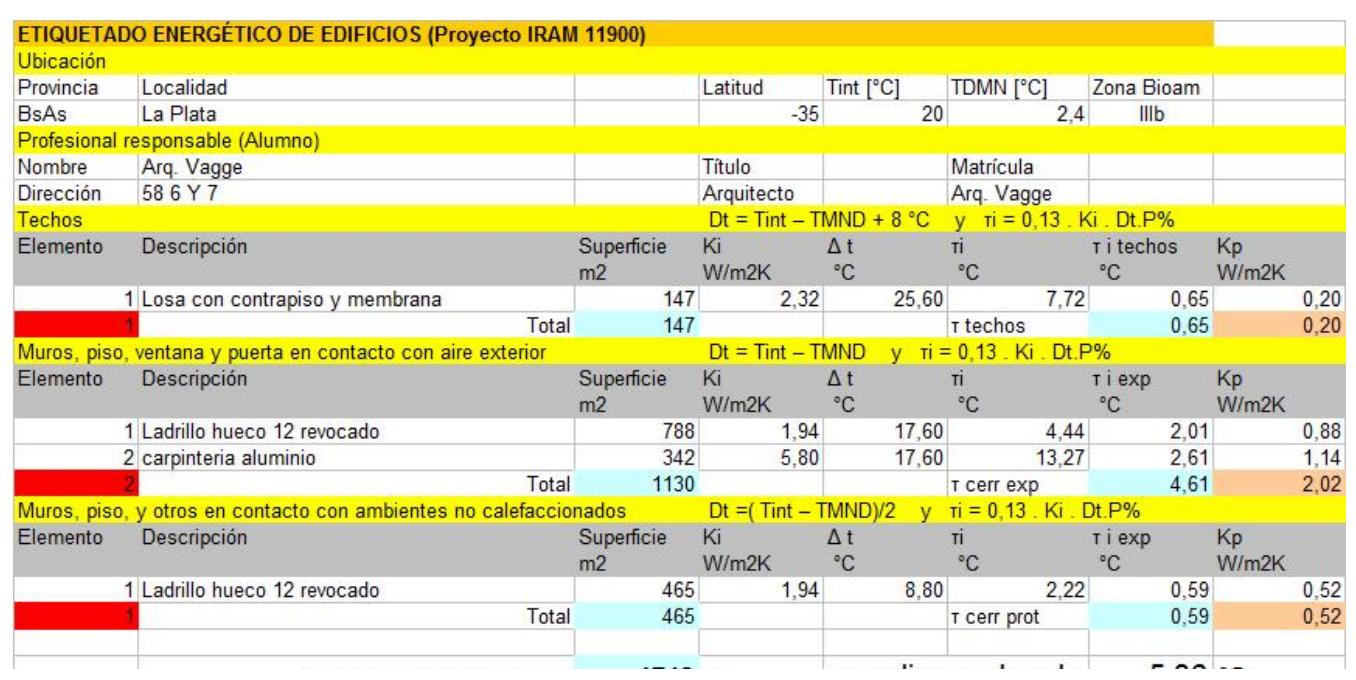

Tabla 4 - Planilla de cálculo

Fonte: Norma IRAM 11900 (INSTITUTO..., 2000c).

\begin{tabular}{|c|c|}
\hline $\begin{array}{l}\text { Clases de } \\
\text { eficiencia } \\
\text { energética }\end{array}$ & Condición ${ }^{1)}$ \\
\hline A & $\tau_{m} \leq 1{ }^{\circ} \mathrm{C}$ \\
\hline$B$ & $1^{\circ} \mathrm{C}<\tau_{m} \leq 1,5^{\circ} \mathrm{C}$ \\
\hline $\mathrm{C}$ & $1,5^{\circ} \mathrm{C}<\tau_{m} \leq 2^{\circ} \mathrm{C}$ \\
\hline$D$ & $2{ }^{\circ} \mathrm{C}<\tau_{m} \leq 2,5^{\circ} \mathrm{C}$ \\
\hline$E$ & $2,5^{\circ} \mathrm{C}<\tau_{m} \leq 3{ }^{\circ} \mathrm{C}$ \\
\hline$F$ & $3^{\circ} \mathrm{C}<\tau_{m} \leq 3,5^{\circ} \mathrm{C}$ \\
\hline G & $3,5^{\circ} \mathrm{C}<\tau_{m} \leq 4{ }^{\circ} \mathrm{C}$ \\
\hline $\mathrm{H}$ & $\tau_{m}>4^{\circ} \mathrm{C}$ \\
\hline
\end{tabular}

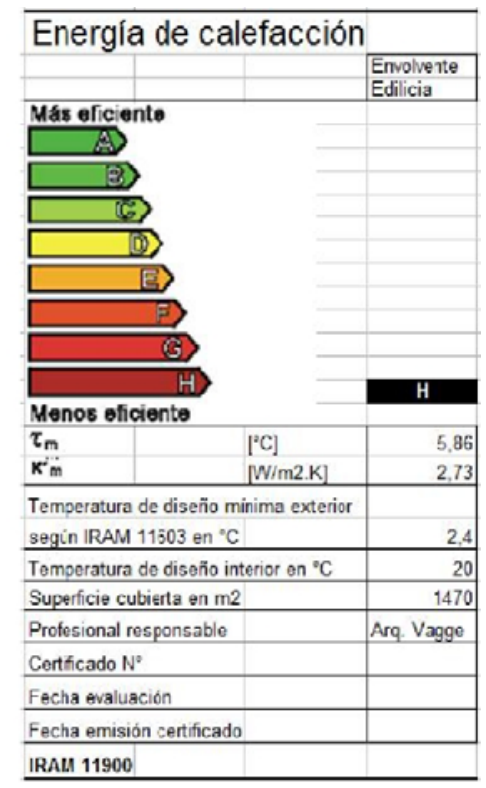

Figura 5 - Etiqueta del modelo de edificio analizado

Fonte: Norma IRAM 11900 (INSTITUTO..., 2000c).
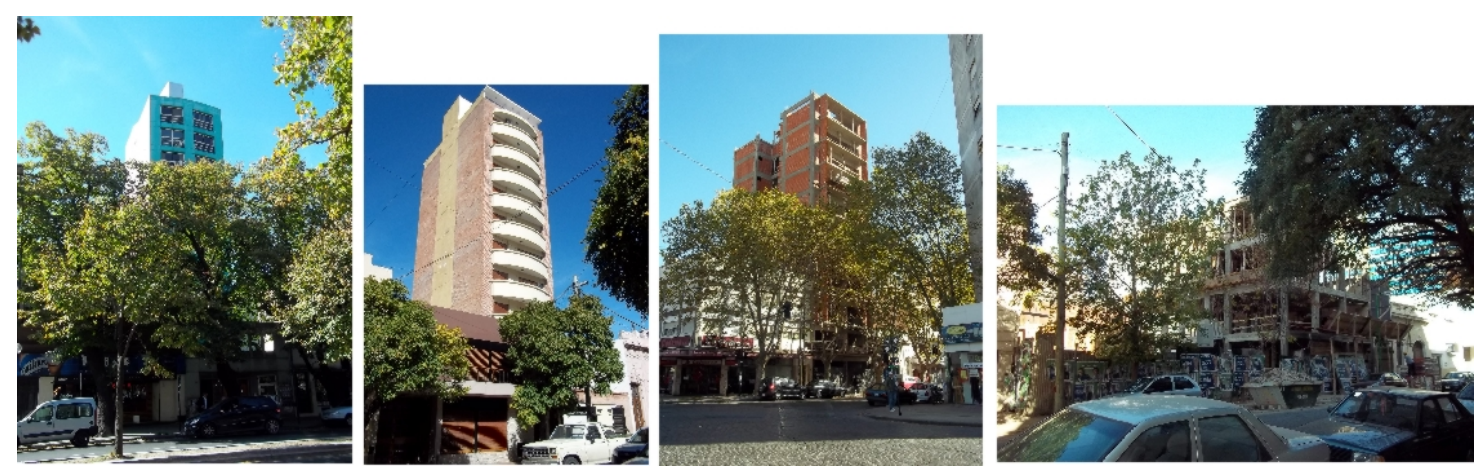

Figura 6 - Registro fotográfico de edificios en la manzana céntrica donde se emplazaría el edificio analizado

32 Vagge, C. S.; Czajkowski, J. D. 

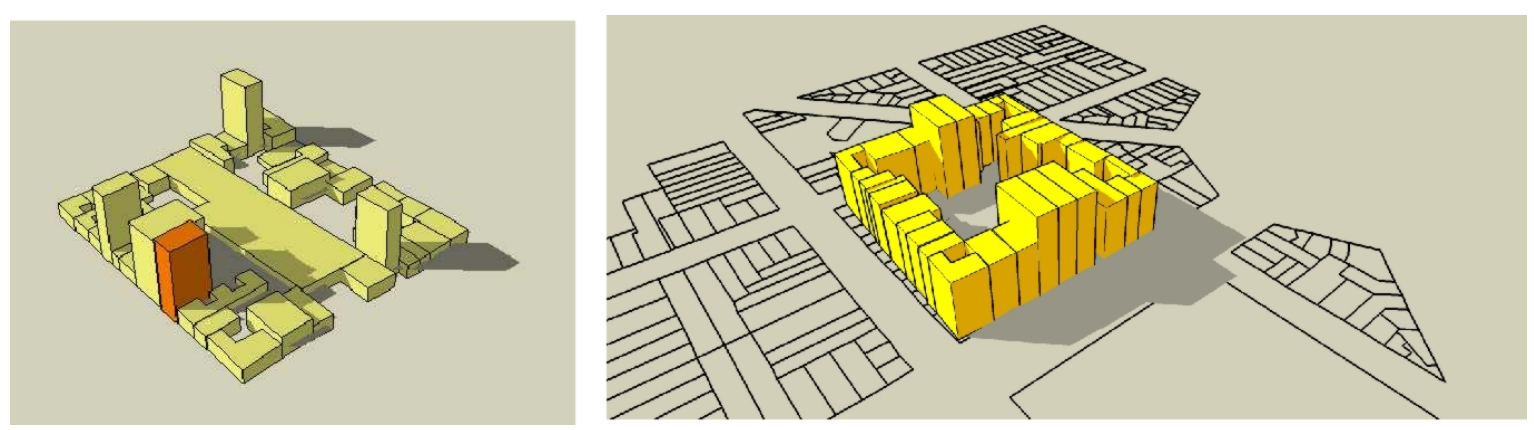

Figura 7 - Volumetría de la situación actual de la manzana donde se encuentra emplazado el modelo de edificio objeto de estudio y su posible evolución hasta alcanzar los 1000 hab/ha
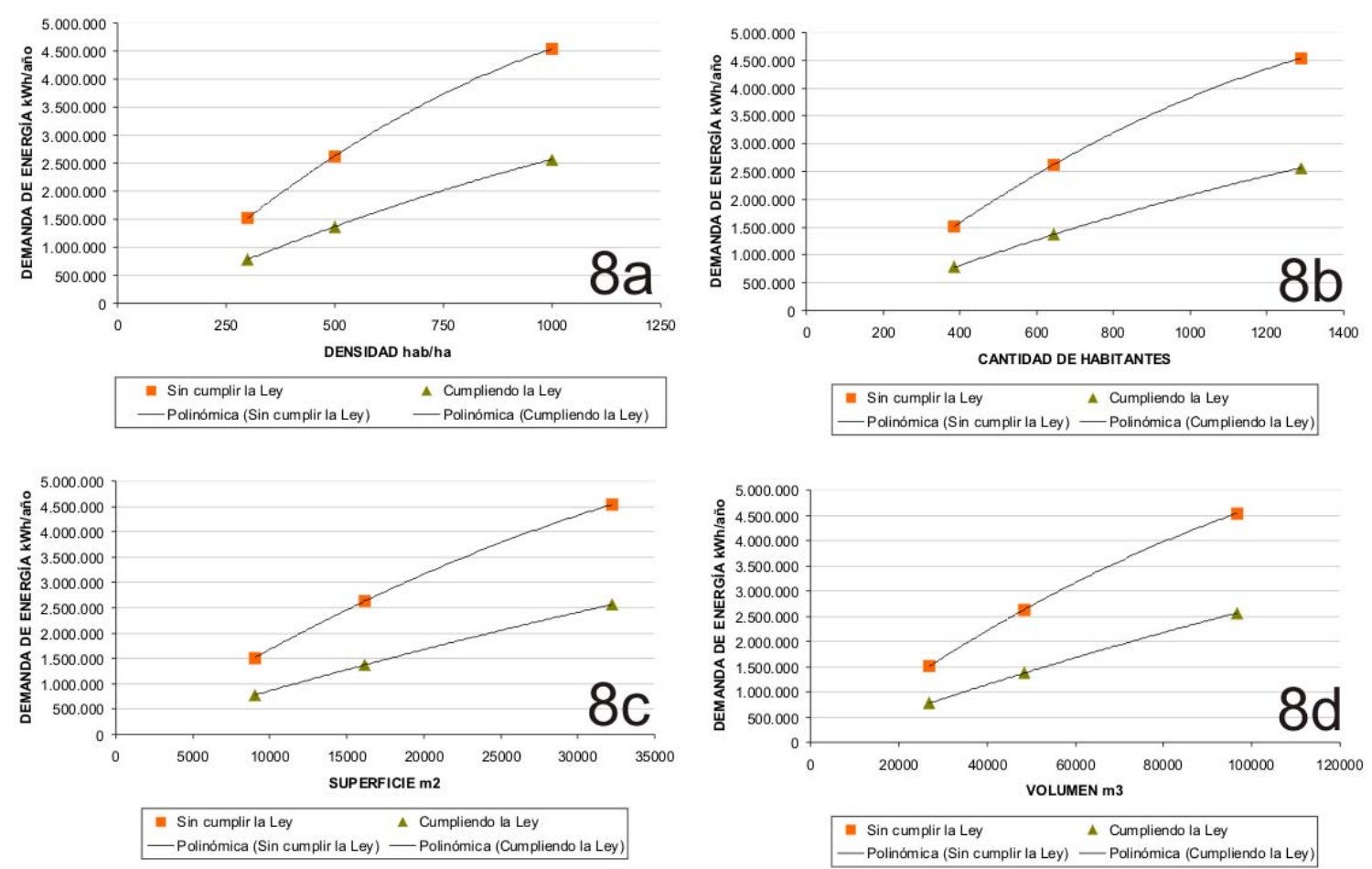

Figura 8 - Gráficos que muestran la demanda de energía, a partir de la evolución de la manzana analizada

En una primera instancia se realizó el cálculo estimativo de la superficie y volumen edificado de la manzana en la actualidad. Se calculó una densidad estimada y luego se determinó una demanda de energía.

Luego se realizaron las mismas estimaciones para un crecimiento de la manzana en un $50 \%$ y luego ya densificada a 1000 hab/ha con la demanda de energía. Con estos datos se realizaron distintos gráficos (Figura 8) para obtener la demanda potencial en calefacción de energía, sin tener en cuenta la Ley, e implementándola. De este modo poder inferir el ahorro potencial de energía.

En la Figura 8 se puede observar los distintos gráficos que muestran en los tres escenarios antes mencionados la relación entre densidad, superficie y volumen construido y demanda de energía. Con las Ecuaciones de la 6 a la 13 de ambas curvas, se integraron con el programa Maple. 15 para conocer el área que representa la demanda de energía sin cumplir la Ley 13059, y cumpliéndola. Luego se calculó la superficie total. De esta manera se pudo determinar que el porcentaje potencial de ahorro de energía en calefacción es de un 46\%; de aplicarse la Ley.

\section{Gráfico 8a}

DAEC $_{\text {ноу }}=-2,4082$ Dens $^{2}+7441,8$ Dens 492926

Ec. 6

DAEC $_{\text {Ley } 13059}=-0,7712$ Dens $^{2}+3549,5$ Dens211935 Ec. 7 
Gráfico $8 \mathrm{~b}$

DAEC $_{\text {HOY }}=-1,4586$ Pers. $^{2}+5791$ Pers. -502474

Ec. 8

DAEC $_{\text {Ley } 13059}=-0,4694$ Pers. $^{2}+2762,9$ Pers.-

216847

Gráfico 8c

DAEC $_{\text {HOY }}=-0,0016 \mathrm{SH}^{2}+197,1 \mathrm{SH}-131167$

DAEC $_{\text {Ley } 13059}=-0,0004 \mathrm{SH}^{2}+92,799 \mathrm{SH}-2635$

Gráfico 8d

DAEC $_{\text {HOY }}=-0,0002 \mathrm{VH}^{2}+65,711 \mathrm{VH}-131518$

$\mathrm{DAEC}_{\text {Ley } 13059}=-4 \mathrm{E}-0,5 \mathrm{VH}^{2}+30,939 \mathrm{VH}-$ 26554

Siendo:

DAEC $_{\text {HOY }}=$ Demanda Anual de energía en Calefacción actual $\left(\mathrm{kWh} / \mathrm{m}^{2}\right)$;

$\mathrm{DAEC}_{\text {Ley }}=$ Demanda Anual de energía en Calefacción implementando la Ley $\left(\mathrm{kWh} / \mathrm{m}^{2}\right)$;

Dens. $=$ Densidad (hab/ha);

Pers. $=$ Personas (adimensional);

$\mathrm{SH}=$ Superficie Habitable $\left(\mathrm{m}^{2}\right) ; \mathrm{y}$

$\mathrm{VH}=$ Volumen Habitable $\left(\mathrm{m}^{3}\right)$.

\section{Consideraciones finales}

El progreso, el desarrollo, el crecimiento, si bien no son términos sinónimos; constituyen las ideas sobre la que se sustentan los cambios que las nuevas planificaciones tienden a avalar. Mientras algunas ciudades planifican su crecimiento basando su competitividad en borrar sus propias señas de identidad cambiándolas por imágenes anodinas descontextualizadas, otras lo hacen a través del desarrollo y potenciación de sus propias características diferenciales. Preservando los valores que han dado sentido trazado, patrimonio ambiental e histórico cultural, escala, paisaje, contacto con la naturaleza, etc. a lo que suman espacios y paisajes propios de la modernidad; estas ciudades, no niegan ni destruyen su identidad cultural. Por el contrario, son capaces de asimilar los cambios y nuevos desafíos sin destruir lo preexistente sino sumando y generando nuevas oportunidades para la ciudad y sus ciudadanos, enriqueciendo aún mas esas señas de identidad de su paisaje urbano consolidado. Del análisis precedente se desprende que es evidente que existe una importante falta de adecuación entre los objetivos teóricos formulados para el desarrollo de la ciudad y las conflictivas estrategias técnicas propuestas para satisfacerlos.

En cuanto a la aplicación de la ley de eficiencia energética al modelo de edificio de construcción usual en La Plata, no verifica sus valores de transmitancia térmica $K$. Tampoco verifica el riesgo de condensación ni el coeficiente global de pérdidas G. Del mismo modo, cuando se aplica el protocolo de etiquetado de eficiencia energética que plantea la norma IRAM 11900 (INSTITUTO..., 2000c), el edifico clasifica como H.

Cuando analizamos la manzana de manera global teniendo en cuenta los valores obtenidos del edificio modelo, en la actualidad, en un escenario cercano contemplando la manzana densificada al $50 \%$ y un escenario a mediano plazo con la manzana densificada al $100 \%$ podemos ver como evolucionará. Podemos además visualizar el crecimiento de demanda de energía y emisiones en función de como se está construyendo hasta el momento y como evolucionaría si se comienza a implementar la Ley de Eficiencia Energética. Se puede ver que el impacto de ahorro potencial en la demanda de energía anual para calefacción sería de un $46 \%$. También en los gráficos se puede observar que a medida que se densifica la manzana, que crece el volumen edificado, las curvas sufren una leve disminución de la pendiente debido a la disminución de superficie expuesta.

Esta situación teniendo en cuenta como esta creciendo la ciudad anticipa consecuencias en la economía nacional basada en subsidios, impactos ambientales negativos y sobre la calidad ambiental futura del medio urbano global.

\section{Referencias}

ARGENTINA. Decreto reglamentario 1030/072010, aprueba la reglamentación de la ley $\mathrm{n}^{\circ}$ 13059 sobre condiciones de acondicionamientos térmico exigibles en la construcción de edificios.

Boletín Oficial de la República Argentina, La Plata, 2 de julio de 2010.

CZAJKOWSKI, J. D.; GÓMEZ, A. Diseño Bioclimático y Economía Energéttica Edilicia. La Plata: UNLP, 2002.

ENVIRONMENT PROTECTION AGENCY.

Greenhouse Gas Equivalencies Calculator.

Disponible:

$<$ http://www.epa.gov/cleanenergy/energyresources/calculator.html>. Acceso: 28 maio 2012. 
ETULAIN, J. C.; LÓPEZ, I. Renovación Edilicia y Transformación Urbana: sustitución tipológica, alteración morfologica y deseconomía urbana, estudio de caso: La Plata-Argentina. Premio Anual de Arquitectura, Urbanismo, Investigación y Teoría. Provincia de Buenos Aires, 1997.

FOSTER, N. Prólogo: arquitectura y sostenibilidad. Revista Tectónica, n. 31, dic. 2010.

GONZALEZ, A. Comparación de Energías y Gases de Efecto Invernadero en Calentamiento de Agua Para Cocción de Alimentos con Electrcidad y Gas Natural. Avances en Energías Renovables y Medio Ambiente, v. 14, p. 7.25-7.32, 2010.

GONZALO, G. Manual de Arquitectura Bioclimática. Tucuman, AR: UNT 1998.

INSTITUTO ARGENTINO DE

NORMALIZACIÓN Y CERTIFICACIÓN.

Norma IRAM $\mathbf{N}^{\circ}$ 11507-1: carpintería de obra y fachadas integrales livianas: ventanas exteriores: parte 1: requisitos básicos y clasificación. Buenos Ayres, 2001a.

INSTITUTO ARGENTINO DE

NORMALIZACIÓN Y CERTIFICACIÓN.

Norma IRAM $N^{\circ}$ 11507-4: carpintería de obra y fachadas integrales livianas: ventanas exteriores: parte 4: requisitos complementarios: aislación térmica. Buenos Ayres, 2001b.

INSTITUTO ARGENTINO DE

NORMALIZACIÓN Y CERTIFICACIÓN.

Norma IRAM No 11601: aislamiento térmico de edificios: propiedades térmicas de los materiales para la construcción: método de cálculo de la resistencia térmica total. Buenos Ayres, 1996a.

INSTITUTO ARGENTINO DE NORMALIZACIÓN Y CERTIFICACIÓN. Norma IRAM No 11604: aislamiento térmico de edifícios: ahorro de energía en calefacción: coeficiente volumétrico $\mathrm{G}$ de pérdidas de calor. Buenos Ayres, 2001c.

\section{INSTITUTO ARGENTINO DE \\ NORMALIZACIÓN Y CERTIFICACIÓN.}

Norma IRAM No 11605: aislamiento térmico de edificios: condiciones de habitabilidad en viviendas: valores máximos admisibles de Transmitancia Térmica "K" (como máximo los valores correspondientes a Nivel B). Buenos Ayres, 1996b.

\section{INSTITUTO ARGENTINO DE}

NORMALIZACIÓN Y CERTIFICACIÓN.

Norma IRAM No 11625: aislamiento térmico de edificios: verificación del riesgo de condensación del vapor de agua superficial e intersticial en paños centrales. Buenos Ayres, 2000a.

\section{INSTITUTO ARGENTINO DE}

NORMALIZACIÓN Y CERTIFICACIÓN.

Norma IRAM No 11630: aislamiento térmico de edificios: verificación riesgo de condensación intersticial y superficial en puntos singulares. Buenos Ayres, 2000b.

\section{INSTITUTO ARGENTINO DE}

NORMALIZACIÓN Y CERTIFICACIÓN.

Norma IRAM $N^{\circ}$ 11900: etiqueta de eficiencia energética de calefacción para edificios:

clasificación según la transmitancia térmica de la envolvente. Buenos Ayres, 2000c.

MUNICIPALIDAD DE LA PLATA. Ordenanza de Ordenamiento Territorial y Uso del Suelo Para el Partido de La Plata. La Plata, 28 de abril de 2010.

SIEMENS. Green Cities. 2011. Disponible em: $<$ http://www.siemens.com/press/pool/de/events/co rporate/2010-11-lam/study-latin-american-greencity-index_spain.pdf>. Acceso: 20 abri. 2011.

\section{Agradecimientos}

A la Profesora Mariana S. Vagge.

Revista Ambiente Construído

Associação Nacional de Tecnologia do Ambiente Construído

Av. Osvaldo Aranha, 99 - 3o andar, Centro

Porto Alegre - RS - Brasil

CEP 90035-190

Telefone: +55 (51) 3308-4084

Fax: +55 (51) 3308-4054

www. seer. ufrgs. br/ ambienteconstruido

E-mail: ambienteconstruido@ufrgs.br 